\title{
Arquitectura hospitalaria y cuidados durante los siglos XV al XIX
}

\section{Hospital architecture and care during the 15 th to the 19th Centuries}

\section{Arquitetura de hospital e cuidados durante a XV ao Século XIX}

Francisco Javier Castro Molina' ${ }^{1}$, Maripaz Castro González², Francisco Megias Lizancos ${ }^{3}$,

Felisa V. Martin Casañas ${ }^{4}$, Alvaro Causapie Castro ${ }^{5}$

${ }^{I}$ Enfermero de Salud Mental del Hospital Universitario de Canarias y Doctorando en Historia del Arte.

${ }^{2}$ Profesora Titular de Universidad, Escuela Universitaria de Ciencias de la Salud, ULE.

${ }^{3}$ Profesor Titular de Universidad, Escuela Universitaria de Enfermería y Fisioterapia, UAH.

${ }^{4}$ Enfermera del Hospital Universitario de Canarias

${ }^{5}$ Licenciado en Historia.

Cómo citar este artículo en edición digital: Castro Molina, F.J; Castro González, MåP; Megias Lizancos, F; Martin Casañas, F.V; Causapie Castro, A. (2012) Arquitectura hospitalaria y cuidados durante los siglos XV al XIX. Cultura de los Cuidados.

(Edición digital) 16, 32. Disponible en: http://dx.doi.org/10.7184/cuid.2012.32.05

Correspondencia: Calle de las Trebinas 24- san Cristóbal de la laguna; 38296-Santa Cruz de Tenerife.

E-mail: javier_tenerife@hotmail.es/teléfono: 626.326.001

Recibido: 11/10/2011 - Aceptado: 20/02/2012

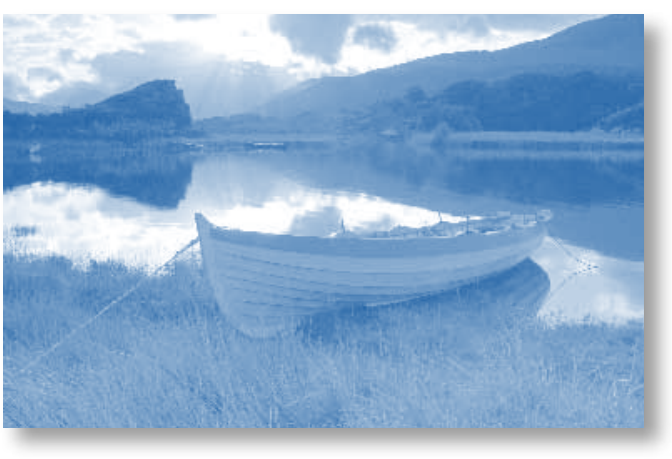

ABSTRACT

This text aims to offer an approach to architecture for healing and care, reviewing the different architectural models, since the fifteenth century have been developed throughout the history of humanity to respond to the needs of health have sprung up to the new examples that arise after their own scientific discoveries of the nineteenth century, a shift in the conception of the hospital building. The brief tour of the hospital architecture, which focuses on the European scene also addresses the importance presented at the time, and has survived until today, the figure of Florence Nightingale, in the Anglo-Saxon environment, such as the rest of the planet.

Keywords: Hospital, hospital architecture, Florence Nightingale, care.

\section{RESUMO}

Este texto destina-se a fornecer uma abordagem de arquitetura para a cura e cuidado, analisando diferentes modelos arquitectónicos, desde o século XV foram desenvolvido ao longo da história da humanidade para dar uma resposta às necessidades de saúde originadas até chegar os novos exemplos que surgem após as descobertas científicas do século XIXproduzindo uma mudança significativa na concepção do edifício hospitalar. A breve turnê da arquitetura do hospital, que concentra-se na cena europeia, também aborda a importância que apresentou no momento, e que nos chegou até agora, a figura de Florence Nightingale, o ambiente de anglo-saxão, ambos no resto do planeta.

Palavras-chave: Hospital, arquitetura hospital, Florence Nightingale, care. 


\section{RESUMEN}

El presente texto pretende ofrecer un acercamiento a la arquitectura para la curación y el cuidado, revisando los diferentes modelos arquitectónicos, que desde el siglo XV se han venido desarrollando a lo largo de la Historia de la Humanidad para poder dar una respuesta a las necesidades de salud que se han originado hasta llegar a los nuevos ejemplos que surgen tras los descubrimientos científicos propios del siglo XIX, produciéndose un giro importante en la concepción del edificio hospitalario. El breve recorrido por la arquitectura hospitalaria, que se centra en el panorama europeo, además aborda la importancia que presentó en su momento, y que nos ha llegado hasta la actualidad, la figura de Florence Nightingale, tanto en el entorno anglosajón, como en el resto del planeta

Palabras clave: Hospital, arquitectura hospitalaria, Florence Nightingale, cuidados.

Las primeras noticias que tenemos acerca de los hospitales, las encontramos en el Imperio Romano de Oriente, en Asia Menor y Siria, lugares donde aparece una fuerte resistencia cristiana, frente al paganismo romano. Ya a mediados del siglo IV, la situación anteriormente comentada, propició la aparición de centros hospitalarios, como el que funda, a las puertas de Cesarea en la Anatolia Oriental, Basilio el Grande (1). Al mismo tiempo, aparecieron otras fundaciones hospitalarias ubicadas en Oriente, los pandokheion (albergues de peregrinos), xenodochium (albergues de forasteros) o los nosocomium (casa de enfermos).

A finales del siglo IV, surgió en Italia y en la Galia merovingia fundaciones hospitalarias, siendo las de mayor relevancia los pertene- cientes a ésta última. Su proliferación, fue debida, en gran medida, al contacto mantenido con el Imperio Bizantino. En el siglo VI, en la Península Ibérica, nos encontramos como el obispo Masona fundó un xenodoquio (580 C.) en la ciudad de Mérida. Es en este momento, cuando aparecen las primeras referencias a hospitales inclusas y casa de lázaros o también llamados casas de leprosos.

Siglos más tarde, durante la Edad Media, proliferaron numerosos establecimientos benéficos, cuya ubicación estaba en el centro de las ciudades, próximas, e incluso anexas, a instituciones eclesiásticas tales como los monasterios, catedrales y conventos. Dos claros ejemplos muestran la realidad del momento: el Hôtel Dieu de París, que data del 829, y el plano ideal, de 820, de la distribución de los diferentes edificios de la abadía de Saint Gall [1].

Desde el siglo XII, tomó relevante importancia este fenómeno asistencial-benéfico por su condición de depositarios de donaciones y legados, que la caridad cristiana consolidaba siglo tras siglo. Destacan excepcionalmente, las órdenes militares, de las que la más antigua fue la de San Juan de Jerusalén u Hospitalarios, presente en el panorama europeo a partir de 1070. Fundaron hospitales en diferentes puntos de la cristiandad, tales como Acre, Villefranche, Viterbo, Chipre, Messina y, sobre todo, Jerusalén, su establecimiento "modélico". Posterior a esta orden, fueron la de los Templarios, fundada en 1118, y la de los Caballeros Teutónicos, fundada en 1190.

Todas estas fundaciones hospitalarias fueron respaldadas por personajes nobles de la sociedad del momento, así como ciudades o villas, e incluso congregaciones religiosas, movidos por una mentalidad donde primaba la caridad en Cristo (2). Por regla general, estos establecimientos benéficos disfrutaban de 
privilegios e inmunidades de la Iglesia, que los eximían de cargas y contribuciones, además del pago por los derechos de alimentos, bebidas y medicinas.

Durante los siglos XII y XIII, los mejores hospitales los podemos encontrar en Francia, tales como el de Angers (fundado en 1153), Ourscamp (Oise, fundado a principios del siglo XIII) y Tonnerre (Yonne, fundado en 1293); el mayor y más famoso, será el Hôtel Dieu de París, compuesto por cuatro grandes salas de dos naves. En el siglo XVII, se desarrollará considerablemente, llegando a dar alojo a 1280 pacientes que en ocasiones precisaban compartir cama con otras personas.

Será, a partir de ahora, una práctica frecuente la separación de los manicomios de los hospitales de agudos; Burdett, cita como ejemplos al de Gante (Bélgica) o el de Bethlehem (Londres) durante los siglos XII y XIII. Esta costumbre no llegará a España hasta el siglo $\mathrm{XV}$, donde ciudades como Valencia, Zaragoza, Sevilla, Valladolid y Toledo, acogerán primeramente esta práctica.

A partir del siglo XV, nos encontramos con un nuevo tipo de estructura hospitalaria que se desarrolló en la península Itálica, siendo el primer ejemplo, el Hospital de Santa Maria Nuova de Florencia fundado en 1286. Ya en 1334 la planta de este establecimiento presenta forma de cruz, con cuatro salas radiales de diferente tamaño (menores las del este y oeste) en cuyo centro estaba ubicado el altar. Esta distribución espacial innovadora, fue un siglo más tarde retomada para la construcción del Pammatone de Génova.

Esta tipología fue trasladada, al que se ha considerado el más importante diseño hospitalario del Renacimiento: el Ospedale $\mathrm{Ma}$ ggiore de Milán [2], encargado por el duque Sforza a Filarete en 1456. Su Treatise, nos lo reproduce y describe de forma detallada: "es un rectángulo de 1000 pies de fachada, dividido en: centro, con gran patio y en medio la capilla; a izquierda y derecha, está constituida por cuatro sala dispuestas en forma de cruz, con su altar en el cruce".

Tanto Santa Maria Nuova como el Ospedale Maggiore constituyeron los modelos de inspiración para el Hospital del Santo Spirito de Sassia, en la reconstrucción que efectuó el Papa Sixto IV entre los años 1474 y 1482.

En Inglaterra y España, esta tipología se hace presente a principio del siglo XVI. Los primeros casos españoles se materializan en los diseños que realizó Enrique de Egas para el Hospital Real de Santiago de Compostela (1501-1511) [3], el Hospital de Santa Cruz de Toledo (1504-1514) y el Hospital Real de Granada (1504). Valencia y Sevilla se unieron a estas nuevas formas durante este mismo siglo.

El Hôpital Saint Louis, diseñado por Claude Vellefaux y fundado por Enrique IV en 1607, fue uno de las mejores edificaciones de su momento, en contraposición con el HôtelDieu, cuya condición de "horrible" no la había perdido desde la Edad Media (3). De manera opuesta, digno de ejemplo fue el Hospital de los Inválidos, obra proyectada por Brunt (4) en 1670, la cual fue comparado con el Hospital de Chelsea, diseñado por el arquitecto anglosajón Wren.

Esta rivalidad entre el mundo galo y anglosajón se hace claramente patente, ante el intento de impulsar como su máximo exponente al Hospital de Greenwich, que se consideró el establecimiento inglés modélico, por excelencia, en este momento.

A finales del siglo XVII y principios del XVIII, vemos como el modelo arquitectónico que había perdurado casi dos siglos, se modificó buscando el aumento de la capacidad 


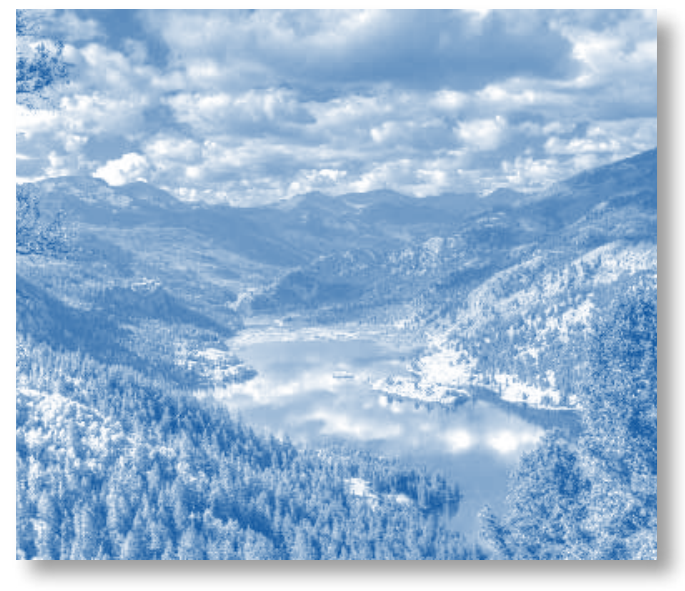

del establecimiento. Antonie Desgodets, en su Edifices antiques de Rome propuso una tipología articulada a partir de un centro octogonal con cerramiento cupular que favoreciera el intercambio de aire con el exterior; en torno a este espacio, disponía dieciséis salas radiales.

Leonhard Christoph Sturm, debió conocer este proyecto durante su estancia en Francia, ya que en 1720 formuló su hospital ideal, en el que, como en el Ospedale Maggiore, el edificio se articula a partir de un rectángulo en el que está inserta en el centro la iglesia de planta octogonal, de la que nacen ocho salas radiales con capacidad para 1525 enfermos (5).

Ya de lleno en el siglo XVIII, la arquitectura hospitalaria presentó un auge considerable, colocándose Gran Bretaña a la cabeza, con innovaciones tales como la especialización por patologías de los pacientes ingresados en los establecimientos. Ejemplos de tal hecho los encontramos en el Hospital de Bethelem (Bedlam) y Saint Luke, destinados a la asistencia a dementes; el Hospital Queen Charlotte, para maternidad; o Foundling que se trataba de una inclusa.

En Norteamérica, los edificios hospitalarios tuvieron impresa la huella anglosajona, remarcándose, como en Gran Bretaña, a principios del siglo XIX, un considerable gusto neoclásico en el que las largas fachadas con pórticos y cúpulas son principalmente su característica más destacada.

Las dimensiones de los establecimientos hospitalarios adquieren un tamaño considerable ante el aumento de la demanda de la población, así como un importantísimo crecimiento de ésta. El Hôtel-Dieu de Lyon, diseño de J.-G. Soufflot en 1741, fue un claro ejemplo de esta situación ante la magnitud de la obra, no viendo su conclusión hasta 1842.

La preocupación por las condiciones higiénicas de los recintos hospitalarios fue marcada a ambos lados del Océano Atlántico. El doctor Rothman, en su examen de los hospitales en norteamericanos, y Chamousset, con su análisis del Hôtel Dieu de París, manifestaron su inquietud ante este tema, al que no se le prestaba la atención necesaria por no considerarlo importante.

Junto a ellos, Jean-Baptiste Le Roy propuso la construcción de pabellones que se articulaban a partir de un patio central, con la intención de favorecer la ventilación de los recintos. Esta idea se materializó en 1756 cuando se dio comienzo al proyecto Rowehead para el Hospital Naval de Stonehouse en Plymouth (6).

A comienzos del siglo XIX, en 1801, Durand en su Recuil et Parallèle comparó gráficamente, tanto el Ospedale Maggiore de Milán como el Pammatone de Génova (7), con ejemplos galos (Saint Louis y Los Incurables, ambos de París) y anglosajones (Stonehouse), además del diseño que realizó Poyet para su segundo informe. Los dos últimos, articulados con pabellones, son los que consideró más adecuado, incorporándolos en su obra titulada, Précis des leçons. En ella, propuso siete pabellones ubicados a ambos lados de un patio central rectangular que moría en un "bosque de columnas" (8). 
La funcionalidad, atendiendo a la distribución espacial de los establecimientos arquitectónicos, primó de manera considerable en Gran Bretaña, aunque se construyeron ejemplos que mostraban concomitancia con modelos anteriores a los acometidos en este momento, muy en la órbita del medievo (9).

En el panorama europeo, esta propuesta se materializó en ejemplos como el Hospital Saint André (1825-1829) de Burdeos de 650 camas, obra de Bourguet; el diseño de Partois para el Hospital de Beaujon en París (1837-1846) y para el de Saint Jean en Bruselas (1838-1849); o Gauthier, que proyectó para la condesa Laribisière un hospital de 905 camas cuyo plano atendía plenamente a la distribución en pabellones [4].

Estos nuevos planteamientos, unidos a la preocupación por la higiene y salubridad de los establecimientos hospitalarios, llevan a tratar la figura de una mujer que en su época marcó un antes y un después en todas las actividades destinadas al cuidado. Guiada por las ideas de progreso de los pensadores victorianos, centró sus esfuerzos en reformar el sistema de cuidados que se prestaban en la salud militar y la elaboración de un programa de formación de enfermeras sólido cimentado por normas profesionales. Para tal empresa se ayudó de la observación y el uso de la estadística social (10).

Florence Nightingale nace en Florencia en 1820 , ciudad en la que su padre, un caballero de buena posición, y el resto la familia, se encontraban durante un viaje efectuado al continente. Recibió una esmerada educación, dominando, con tan solo 17 años, varios idiomas antiguos y modernos, además de diferentes disciplinas propias de una señorita de la alta sociedad victoriana.

Tras cumplir los veinte años, mostró un marcado interés por el cuidado de los enfermos y las condiciones físicas de los hospitales, situación que le llevó a visitar varios establecimientos con la intención de conocer sus condiciones y organización.

En 1847, marchó a Alemania durante varios meses, tras haber visitado con anterioridad Egipto y Grecia, lugares donde se familiarizó con la práctica del cuidado de los enfermos realizada por las órdenes religiosas. En el mundo germano, se formó con las Diaconisas de Kaiserswerth (11), para posteriormente viajar a París, ciudad donde recibió estudios de las Hermanas de la Caridad en la Maison de la Providence.

Un año más tarde, en 1854, Florence se desplaza a Crimea para la dirección y coordinación de un hospital turco (12). El inicio de esta Guerra llevó a que el Secretario de Guerra, Sir Sydney Herbert, solicitara su auxilio, junto a treinta y siete enfermeras más (13).

Florence Nightingale fue nombrada superintendente del Female Nursing Establishment of the English General Hospitals de Turquía. Este hospital militar, de planta cuadrada, con capacidad para albergar a 1700 pacientes, alojaba en realidad, un total de casi 4000 enfermos. Los cuidados prestados a los soldados ingresados en el establecimiento eran conside-

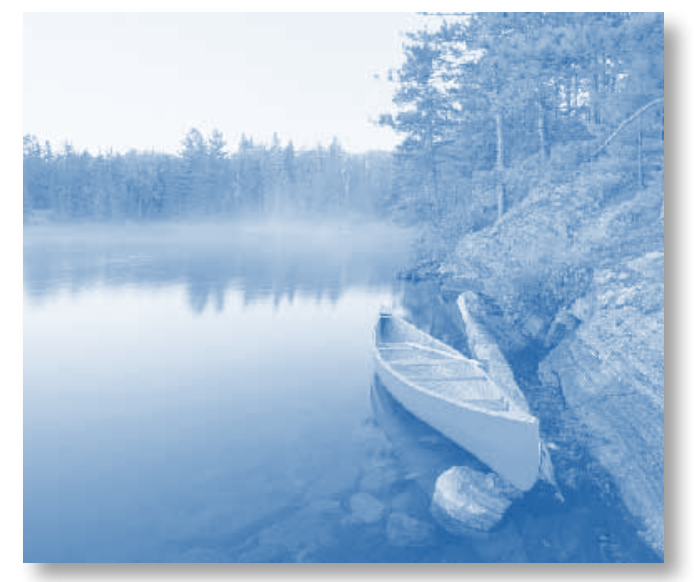


rablemente precarios, llegándolos a calificar de “vergonzosos" (14).

En 1856 regresó a casa, débil, tras haber estado gravemente enferma de la "fiebre de Crimea”, situación que casi la llevó al borde de la muerte. A partir de este momento, su interés se centró en la mejora de las condiciones de los hospitales ingleses, fijando su vista en el Hospital Militar de Netley, en Hampshire, establecimiento comenzado en 1856, para el que propuso el proyecto diseñado para Lariboisière.

Este mismo año, un médico de Edimburgo llamado John Roberton, manifestó públicamente su apoyo a los establecimientos hospitalarios articulados a partir de pabellones (15), situación que interesó considerablemente a Florence.

Lord Panmure remitió el proyecto de Netley a Florence Nightingale para que se pronunciara; ella apeló a Palmerston con argumentos que lo convencieron, aunque el edificio continuó construyéndose, sin modificación alguna, hasta su conclusión en 1863 (16).

En los siguientes años, realizó numerosas publicaciones con las que procuró dar una visión antagónica, a la que se tenía en ese momento, sobre las condiciones higiénicas de los hospitales y su destacada importancia para la rápida y correcta reposición de los enfermos que en ellos se alojaban. Entre ellas destacaron obras tales como: Notas sobre las cuestiones relativas a la salud, eficacia y administración hospitalaria del ejercito británico (1858); Notas de Hospitales (1859); Observaciones sobre la situación sanitaria del ejército en la India (1863); ¿Vida o muerte en la India? (1873); Notas de enfermería para las clases trabajadoras (1861); y su obra más universal, Notas sobre enfermería: qué es y qué no es (1859), donde plasmó su filosofía entorno a los cuidados, conductas y las condiciones higiénicas que deben estar presentes en un hospital (17). A todo este arduo trabajo, se le unió la elaboración de una nomenclatura estandarizada para las enfermedades y el diseño de un Formato Estadístico del Hospital Modelo (18).

La arquitectura hospitalaria articulada a partir de pabellones fue argumentada y defendida por diferentes autores, entre ellos Kuhn en su Handbuch der Architektur (1897) o Guadet en Elèments et thèoriesde l'architecture (1902). Esta propuesta arquitectónica se convirtió en un "signo de autoridad progresista". Los ejemplos son múltiples, entre ellos, el ya nombrado de Lariboisière y el nuevo Hôtel-Dieu de París, reformado tras su incendio de 1772 .

Numerosas veces fue consultada Miss Nightingale para la planificación de nuevos hospitales en Inglaterra, Australia, Canadá y los Estados Unidos de Norteamérica, destacando, en este último país, la crítica que efectuó a los planos del Johns Hopkins Hospital de Baltimore, obra de Niernsee que se concluyó en 1879, tres años después de su comienzo.

Otro importante avance acaecido durante el siglo XIX fue la especialización de los establecimientos hospitalarios para mejorar los cuidados prestados a los pacientes, atendiendo a un solo tipo de dolencia. De esta manera se crearon los hospitales-inclusas, asilos para dementes, hospitales para pernoctar,... Tempranamente, durante el primer tercio de este siglo, encontramos los primeros ejemplos en Londres y París.

En España, el Real Decreto de 1885 refleja la concepción que la sociedad de la época tenía del enfermo mental como una especie de ser extraño y temible, poseído o endemoniado, de cuya presencia había que proteger al cuerpo social por medio de la reclusión. El ingreso en el recinto hospitalario, el manicomio, constituyó en aquel tiempo la atribución por parte 
de la sociedad de la condición de loco a un individuo, situación que conllevó a establecer importantes trabas legales para evitar ingresos improcedentes, aunque una vez producido éste, las expectativas de salida de los manicomios eran casi nulas. La Ley de Beneficencia de 1849 recogía la obligación del Estado de proporcionar atención sanitaria a sus ciudadanos, si bien las Diputaciones fueron asumiendo esta responsabilidad en el campo de la salud mental iniciando la construcción de manicomios, hasta que un decreto de 19 de abril de 1887 estableció que la obligación de atender a los dementes era imputable a la Diputación de cada provincia.

Aunque la legislación vigente acotaba estas cuestiones, la inexistencia de recursos asistenciales fue la tónica presente durante el siglo XIX, no recibiendo los alienados atención sanitaria y encontrándose desatendidos por las calles o encerrados en sombríos calabozos en los que transcurrían sus días. Los manicomios de la época eran verdaderos pudrideros de locos en los que el ambiente reinante, lejos de favorecer la buena evolución de los pacientes, contribuía a su descompensación y a su desorganización.

Ante la ausencia de hospitales psiquiátricos en el País Vasco, los enfermos eran ingresados fundamentalmente en los Manicomios de Valladolid y Zaragoza, lo que implicaba un importante desplazamiento y en muchos casos, una definitiva desconexión de la comunidad originaria.

El siglo XX vendrá marcado, considerablemente, por una sucesión de formas estéticas que se remarcan en esta tipología. Además de los pabellones, aparecen las colonias que procuraron ubicar los recintos sanitarios en zonas rurales, situación que marcó una diferencia con respecto al siglo XIX. El primer ejemplo de esta innovación arquitectónica se acometió en la ciudad de Nancy, en el Hospital de Maréville.

En los años sucesivos a la década de 1870 , el concepto de los hospitales en pabellones se fue desechando poco a poco, para dar paso a formas arquitectónicas que se elevaban, creciendo en altura. Estas nuevas soluciones se debieron principalmente a las novedades que aparecieron en este momento, con respecto a materiales y técnicas constructivas, a lo que se unió nuevos descubrimientos en el campo de la medicina que efectuaron los doctores Luis Pasteur y Joseph Lister (19). Estos nuevos hallazgos, derogaban el anterior concepto de mediados del XIX en el que el aire enrarecido era el principal enemigo de los pacientes enfermos hospitalizados.

En 1907, Ochsner y Sturm (20) defienden el "compactar edificios de pisos" como solución a las necesidades de la arquitectura hospitalaria. Esta compactación buscaba disminuir la movilidad del paciente y minimizando su contacto con el exterior. Los primeros ejemplos están presentes en Estados Unidos de Norteamérica, como el que diseñó James Gamble Rogers para el Columbia Presbyterian Medical Center de Nueva York, con una capacidad total de 1499 camas.

\section{BIBLIOGRAFÍA}

- Arrechea Miguel, J. (1989). Arquitectura y Romanticismo. El pensamiento arquitectónico en la España del siglo XIX. Secretariado de publicaciones de la Universidad de Valladolid. Valladolid.

- Castiglioni, A. (1941). Historia de la medicina. Editorial Salvat. Barcelona.

- Clavijo Hernández, F. (1982). El Hospital de San Salvador de Sevilla en el siglo XV. III Coloquio de Historia Medieval Andaluza. Jaén.

- Cohen L.B. (1984). Florence Nightingale. Scientific American.s.l. 
- Diepgen, P. (1925). Historia de la medicina. Editorial Labor. Barcelona.

- Donahue, M. P. (1988). Historia de la Enfermería. Ediciones Doyma. Barcelona.

- García Melero, J. E. (1998). Arte español de la Ilustración y del siglo XIX: en torno a la imagen del pasado. Encuentro L. Madrid.

- García Melero, J. E; Viñuelas González, J. M. (1996). Historia del arte español moderno y contemporáneo.; Universidad Nacional de Educación a Distancia (UNED); Madrid.

- García Melero, J. E.; Bastida de la Calle, M. D. (1996). La obsesión por la imagen del pasado: el romanticismo, sus orígenes clasicistas y su incidencia en las vanguardias artísticas; Universidad Nacional de Educación a Distancia (UNED); Madrid.

- Guerra, F. (1982). Historia de la medicina. Editorial Norma. Madrid.

- Granel, L. S. (1980). Historia de los hospitales en el siglo XVIII. s. e. Valladolid.

- Hernández Conesa, J. (1995). Historia de la Enfermería: un análisis histórico de los cuidados de enfermería. Interamericana McGraw-Hill. Madrid.

- López Piñero, J. M. (2000). Breve historia de la medicina. Editorial Alianza S. L. Madrid.

- Nightingale, F. (1999). Notas sobre Enfermería. Qué es y qué no es. Editorial Masson. Barcelona.

- Palacios Bañuelos, L. (2003). Manual de Historia Contemporánea Universal. Primera edición. Ed. Dykinson. Barcelona.

- Pevsner, N. (1979): Historia tipológica arquitectónica. Editorial Gustavo Gili. Barcelona.

- Riera, J. (1975). Planos de los Hospitales españoles del siglo XVIII existentes en el Archivo General de Simancas. Seminario de Historia de la Universidad de Valladolid. Valladolid.

- Rodríguez Maffiote, C. (1981). Historia de la medicina. Conrado Rodríguez Maffiote S.L. Santa Cruz de Tenerife.

- Santamaria Almolda, R. (2000): La tipología hospitalaria española en la Real Academia de las Bellas Artes de San Fernando (1814-1875). Tesis dirigida por Dr. José Enri- que García Melero; Publicaciones de la UNED. Madrid. - Sutcliffe, J. (1993). Breve historia de la medicina: desde la prehistoria hasta el año 2020. Editorial Blume. Barcelona.

- Vidler, A. (1997). El espacio de la Ilustración. Alianza Forma. Madrid.

- Woodham-Smith, C. (1951): Florence Nightingale. Ediciones McGraw-Hill Book Co. Nueva York.

\section{NOTAS:}

(1) Fue fundado en el 370 a.C., destinado a los enfermos, siendo sus benefactores los poderosos del lugar.

(2) Clavijo Hernández, F. (1982). El Hospital de San Salvador de Sevilla en el siglo XV. III Coloquio de Historia Medieval Andaluza. Jaén pp. 78-83.

(3) Pese a que había sido remodelado también por Claude Vellefaux.

(4) Su capilla, la de Saint Louis-des-Invalides, fue diseñada por Hardouin-Mansart.

(5) Esta planta en forma de cruz estará presente hasta el siglo XIX, donde será de primera elección en los diseños propuestos para asilos.

(6) Esta solución arquitectónica está presente en las obras de Howard, Lazaretos (1789) y la de Durand, Recueil, donde Stonehouse es reproducida y tomada como ejemplo.

(7) Ambos con una distribución en forma de cruz.

(8) Esta solución recuerda a las propuestas por Boulleè a modo de ornato.

(9) Stonehouse y el proyecto de Stonehouse-Le Roy-Tenon se utilizó en casos excepcionales.

(10) Cohen L.B. (1984). Florence Nightingale. Scientific American. s.l. p.128.

En 1853, asumió la superintendencia del Establishment for Gentlewomen During Illness de Londres. Su administración y gestión fue modélica, procurando incluso crear una escuela para la formación de enfermeras. Nunca se llevó a cabo tal empresa, lo que decepcionó considerablemente a Florence.

(11) Las Diaconisas de Kaiserswerth se fundan hacia 1833 a iniciativa de Teodor Fliedner (1800-1864). 
(12) Woodham-Smith, C. (1951). Florence Nightingale. Ediciones McGraw-Hill Book Co. Nueva York pp. 87-89.

(13) Donahue, M. P. (1988). Historia de la Enfermería. Editorial Doyma. Barcelona pp. 242-243.

(14) Ibídem; pp. 242-243.

Ante esta precaria situación, William Howard Russell, corresponsal de guerra del periódico The Times remitió varias crónicas que denunciaban la realidad sanitaria de este conflicto bélico . Florence Nightingale tomó medidas con respecto a la higiene y de organización que consiguieron disminuir la mortalidad de manera sorprendente, de un 42 a un $2.2 \%$.

(15) Pone como ejemplos los hospitales de: Saint André en Burdeos, Saint Jean en Bruselas y Beaujon y Lariboisière en París.

(16) Pevsner, N (1979). Historia tipológica arquitectónica. Editorial Gustavo Gili. Barcelona p.185.

(17) Ibídem; pp. 134-140.

(18) Aprobado por el Congreso Internacional de Estadística celebrado en Londres en 1860.

(19) El Dr. Luis Pasteur descubrió que las bacterias eran los agentes causantes de la enfermedad; el Dr. Joseph Lister formuló la importancia del tratamiento antiséptico de las heridas para evitar infecciones.

(20) Pevsner, N. (1979): opus cit; p.186.

\section{ANEXOS FOTOGRÁFICOS}

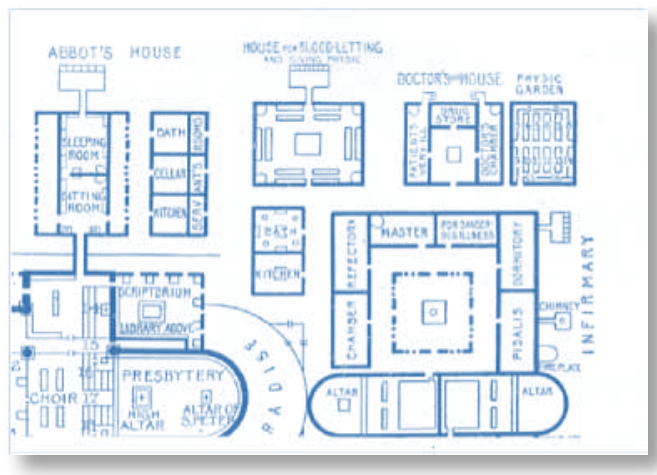

[1] Plano ideal para la abadía de Sant Gall, aproximadamente del 820: detalle de la enfermería.

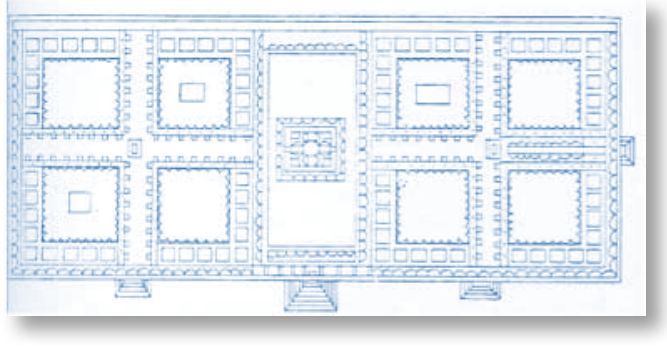

[2] Filarete, plano propuesto para el Ospedale Maggiore de Milán (posterior a su Treatise, comienzos de la década de 1460)

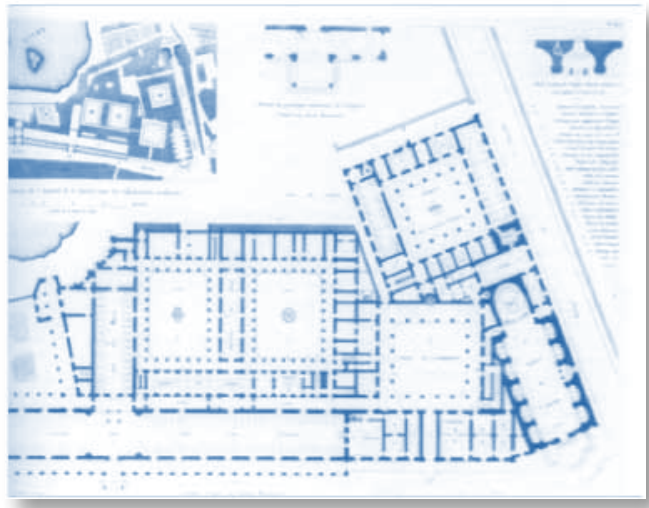

[3] Santiago de Compostela, Hospital Real, 1501-1511, de Enrique Egas: plano.
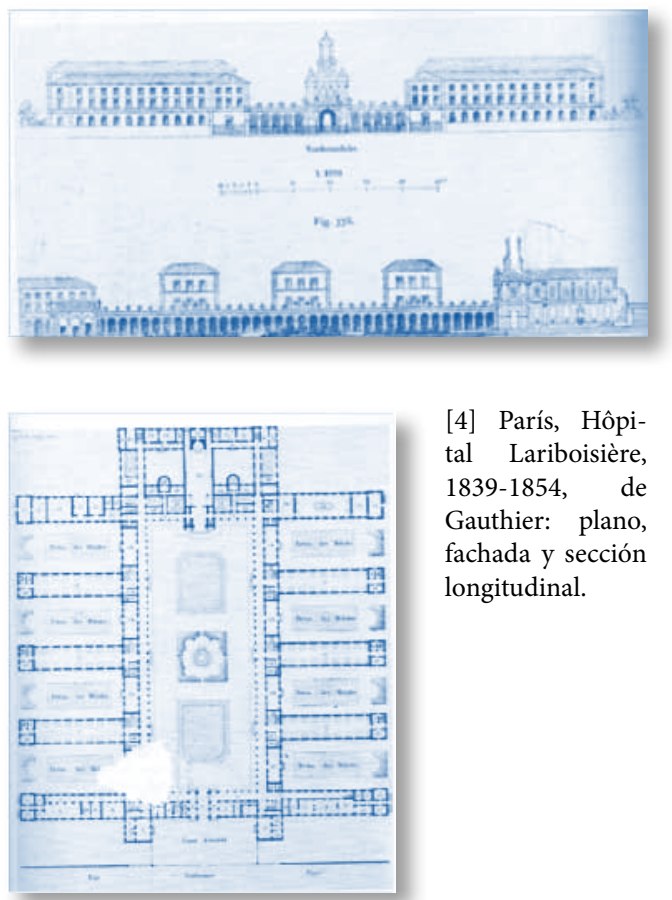
[4] París, Hôpital Lariboisière, 1839-1854, de Gauthier: plano, fachada y sección longitudinal. 